\title{
Anti-mitochondrial antibodies in patients with Graves' disease may not signify primary biliary cirrhosis
}

\author{
P. Perros, J.M. Palmer ${ }^{1}$, S.J. Yeaman ${ }^{1}$ and P. Kendall-Taylor
}

Departments of Medicine, and ${ }^{1}$ Biochemistry and Genetics, Medical School, University of Newcastle upon Tyne, Newcastle upon Tyne NE2 4HH, UK

\begin{abstract}
Summary: Two patients with Graves' disease were incidentally found to have anti-mitochondrial antibodies by immunofluorescence in the absence of symptoms, clinical signs or biochemical evidence of liver dysfunction. Anti-mitochondrial antibody titres became undetectable in both patients on follow-up. Screening of the patients' sera by immunoblotting against the purified antigens of the M2 complex was negative. We conclude that in these cases, anti-mitochondrial antibodies detected by immunofluorescence were directed against antigens other than the primary biliary cirrhosis-associated M2 complex and therefore did not signify subclinical primary biliary cirrhosis.
\end{abstract}

\section{Introduction}

Anti-mitochondial antibodies are found in over $95 \%$ of patients with primary biliary cirrhosis and are routinely detected by immunofluorescence. ${ }^{1}$ The mitochondrial antigens are components of the mitochondrial 2-oxo acid dehydrogenase complex (M2 complex), and antibodies to these antigens can be detected by immunoblotting. ${ }^{2}$ Two patients with Graves' disease and positive anti-mitochondrial antibodies were identified. Neither patient had evidence of liver disease which prompted us to follow their serum titres to anti-mitochondrial antibodies serially and study their antibody reactivity to the purified antigens.

\section{Materials and methods}

Anti-mitochondrial antibodies were measured by immunofluorescence and by immunoblotting as described previously. ${ }^{2}$

\section{Results}

Patient 1 was a 14 year old female with Graves' disease. Anti-mitochondrial antibodies were absent at presentation, present at 2 years (titre 1:40), 4 years (titre $1: 20$ ) but absent at 6 years.

Patient 2 was a 48 year old female with Graves' disease. Anti-mitochondrial antibodies were detec-

Correspondence: P. Perros, B.Sc., M.B.B.S., M.R.C.P., M.D., Department of Diabetes, Royal Infirmary, Edinburgh EH3 9YW, UK

Accepted: 9 August 1993 table at presentation (titre 1:40), 2 years $(1: 320)$ and 4 years (1:40), but not at 6 years.

Both patients had normal liver function tests and IgM levels at all times. Antibody reactivity against

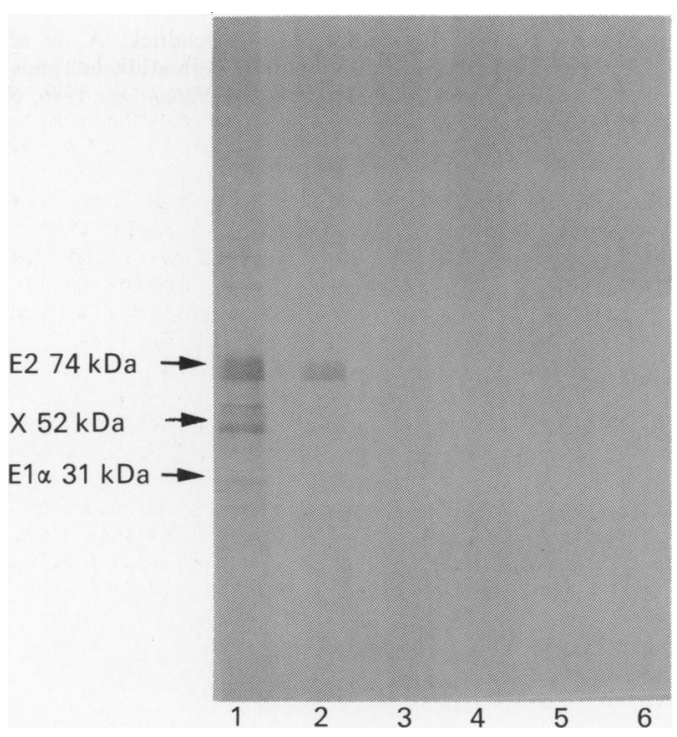

Figure 1 Immunoblots of sera against the purified mitochondrial M2 complex. Patients 1 and 2 failed to recognize any of the subunits of $M 2$ even in supraoptimal dilutions (1:100). E2, $X$, and $E 1 \alpha$ are subunits of the M2 complex. Lanes 1 and 2: positive control sera of patients with primary biliary cirrhosis (1:1,000 dilution). Lane 3 : patient 1 (1:100 dilution). Lane 4: patient 2 (1:100 dilution). Lane 5 : negative control serum of a patient with chronic active hepatitis (1:100 dilution). Lane 6: negative normal control serum (1:100 dilution). 
the purified M2 complex by immunoblotting was absent in both patients' sera (Figure 1).

\section{Discussion}

Anti-mitochondrial antibodies are detected routinely by immunofluorescence, which is highly specific for identifying patients with primary biliary cirrhosis. ${ }^{1}$ Patients with primary biliary cirrhosis whose sera are positive for anti-mitochondrial antibodies by immunofluorescence but negative against the $\mathbf{M} 2$ complex by immunoblotting are extremely rare, and usually associated with abnormal serum biochemical liver tests. ${ }^{3}$ The absence of liver dysfunction in the cases reported here, combined with the lack of serum reactivity against the M2 antigens by immunoblotting, suggests that they did not have primary biliary cirrhosis. Patients with autoimmune thyroid disease may produce anti- bodies to the mitochondrial ATP/ADP transporter protein, ${ }^{4}$ and it is possible that our patients possessed such antibodies.

Primary biliary cirrhosis is associated with autoimmune thyroid disease, and anti-mitochondrial antibodies in patients with Graves' disease have been described. ${ }^{5}$ However, positive anti-mitochondrial antibodies by immunofluorescence in patients with autoimmune thyroid disease do not necessarily signify the presence of subclinical primary biliary cirrhosis. Testing such sera against the M2 antigens by immunoblotting may be a useful means of resolving this problem.

\section{Acknowledgement}

The authors wish to thank Professor O.F.W. James $\vec{\nabla}$ for constructive comments.

\section{References}

1. Kaplan, M.M. Primary biliary cirrhosis. N Engl J Med 1987, 316: $521-528$.

2. Fussey, S.P.M., Guest, J.R., James, O.F.W., Bassendine, M.F. \& Yeaman, S.J. Identification and analysis of the major M2 antigens in primary biliary cirrhosis. Proc Natl Acad Sci USA 1988, 85: 8654-8658.

3. Mitchison, H.C., Bassendine, M.F., Hendrick, A. et al. Positive antimitochondrial antibody but normal alkaline phosphatase: is it primary biliary cirrhosis? Hepatology 1986, 6: $1279-1284$.

4. Zarrilli, R., Oates, E.L., McBride, O.W. et al. Sequence and chromosomal assignment of a novel cDNA identified by immunoscreening of a thyroid expression library: similarity to a family of mitochondrial solute carrier proteins. Mol Endocrinol 1989, 3: $1498-1508$.

5. Heseltine, L., Turner, I.B., Fussey, S.P.M. et al. Primary biliary cirrhosis: quantitation of autoantibodies to purified mitochondrial enzymes and correlation with disease progression. Gastroenterology 1990, 99: 1786-1792. 